\title{
Coronary artery disease in Alström syndrome
}

\author{
Kumar Jatti ${ }^{\star, 1}$, Richard Paisey ${ }^{2}$ and Ranjit More ${ }^{1}$ \\ Alström syndrome (ALMS) is a rare autosomal recessive condition, caused by mutations in the ALMS1 gene located on the short \\ arm of chromosome 2. This gene codes for a protein linked with the centrosome, whose precise function is unknown. This \\ condition was first described by Alström in 1959. ALMS is a multisystem condition that is characterised by childhood onset of \\ blindness secondary to rod-cone retinal degeneration and dilated cardiomyopathy with heart failure, which often presents in \\ infanthood and may recur later in life. Metabolic abnormalities including hypertriglyceridemia, liver steatosis, insulin resistance \\ and type 2 diabetes mellitus are common, often occurring in association with obesity. Other abnormalities include \\ endocrinological disturbances, such as thyroid disorder, growth hormone deficiency, hypogonadism and, in women, \\ hyperandrogenism. This syndrome is also associated with sensorineural hearing loss, renal failure secondary to glomerulo- \\ fibrosis, and fibrotic lung disease. Multiorgan fibrotic infiltration is the common feature in all cases. Considering the history of \\ diabetes, hypertension, dyslipidemia, obesity and renal dysfunction in ALMS, it would be expected that this group of patients \\ could develop coronary artery disease (CAD). But such cases have not been reported so far. We report a case of premature onset \\ of CAD in one of the longest surviving patient with ALMS. \\ European Journal of Human Genetics (2012) 20, 117-118; doi:10.1038/ejhg.2011.168; published online 7 September 2011
}

Keywords: Alström syndrome; complications; coronary artery disease

\section{INTRODUCTION}

Alström syndrome (ALMS) is a rare autosomal recessive condition, caused by mutations in the ALMS1 gene located on the short arm of chromosome 2. This gene codes for a protein linked with the centrosome, whose precise function is unknown. This condition was first described by Alström in $1959 .{ }^{1}$ ALMS is a multisystem condition that is characterised by childhood onset of blindness secondary to rod-cone retinal degeneration and dilated cardiomyopathy with heart failure, which often presents in infanthood and may recur later in life. ${ }^{2}$ Metabolic abnormalities including hypertriglyceridemia, liver steatosis, insulin resistance and type 2 diabetes mellitus are common, often occurring in association with obesity. ${ }^{3-5}$ Other abnormalities include endocrinological disturbances, such as thyroid disorder, growth hormone deficiency, hypogonadism and, in women, hyperandrogenism. This syndrome is also associated with sensorineural hearing loss, renal failure secondary to glomerulo-fibrosis, and fibrotic lung disease. ${ }^{2}$ Multiorgan fibrotic infiltration is the common feature in all cases.

Considering the history of diabetes, hypertension, dyslipidemia, obesity and renal dysfunction in ALMS, it would be expected that this group of patients could develop coronary artery disease (CAD). But such cases have not been reported so far. We report a case of premature onset of $\mathrm{CAD}$ in one of the longest surviving patient with ALMS.

\section{CASE PRESENTATION}

A 40-year-old ALMS male patient with diabetes, hypertension, blindness, sensorineural deafness and chronic renal impairment, presented with a history of exertional chest tightness and shortness of breath. $\mathrm{He}$ was investigated initially with treadmill exercise testing, which was positive and transthoracic echocardiography demonstrated mild concentric left ventricular hypertrophy with good systolic function.
Our case thus had no features of dilated cardiomyopathy. Coronary angiography (see Figure 1) demonstrated significant stenotic disease in the proximal portion of the left anterior descending artery (LAD). However, the circumflex and right coronary arteries were free of stenotic disease and he was listed for elective angioplasty to his LAD artery lesion. Taking into account his chronic renal impairment (creatinine of $278 \mathrm{mmol} / \mathrm{l}$ ), angioplasty was carried out with IV fluids and $\mathrm{N}$-acetyl cysteine peri-procedurally as per local renal protocol. Angioplasty was initially attempted using a right radial artery approach. It was abandoned because of severe angulation of the innominate artery at its entry to the aortic arch, and therefore the procedure was carried out via a right femoral approach. He had successful angioplasty with deployment of a drug-eluting stent (Promus $2.5 / 18 \mathrm{~mm}$ ) to the proximal LAD artery lesion, which resulted in good distal flow (see Figure 2). The procedure was uneventful and the femoral artery puncture site was sealed with an angioseal. He was discharged the next day, after overnight observation. Angioplasty produced good symptomatic relief of his anginal symptoms and there was a significant improvement in his exercise capacity.

\section{DISCUSSION}

Cardiomyopathy is a well-described complication in ALMS. However, symptomatic CAD has been predicted possibly because of associated diabetes, hypertension, dyslipidemia, obesity and renal impairment, ${ }^{6}$ but it has not been reported so far in the literature.

The aetiology and pathophysiology underlying cardiomyopathy in ALMS are incompletely understood. Echocardiography studies have reported dilated cardiomyopathy, ${ }^{7}$ whereas smaller studies with tissue Doppler have reported restrictive pattern. ${ }^{8}$ A small autopsy series (five patients) demonstrated myocardial fibrosis. ${ }^{3}$ Frequent coexistence of obesity, insulin resistance, glucose intolerance, hypertension,

\footnotetext{
${ }^{1}$ Department of Cardiology, Lancashire Cardiac Centre, Blackpool Victoria Hospital, Blackpool, UK; ${ }^{2}$ Department of Endocrinology and Diabetes, Torbay Hospital, Devon, UK ${ }^{*}$ Correspondence: Dr K Jatti, Lancashire cardiac centre, Blackpool Victoria Hospital, Whinney Heys Road, 1, West Green Close, Edgbaston, Birmingham B15 2LA, UK. Tel: +44 781754 3767; Fax: +44 121218 0518; E-mail: kumarjatti@yahoo.com

Received 18 January 2011; revised 2 May 2011; accepted 2 August 2011; published online 7 September 2011
} 


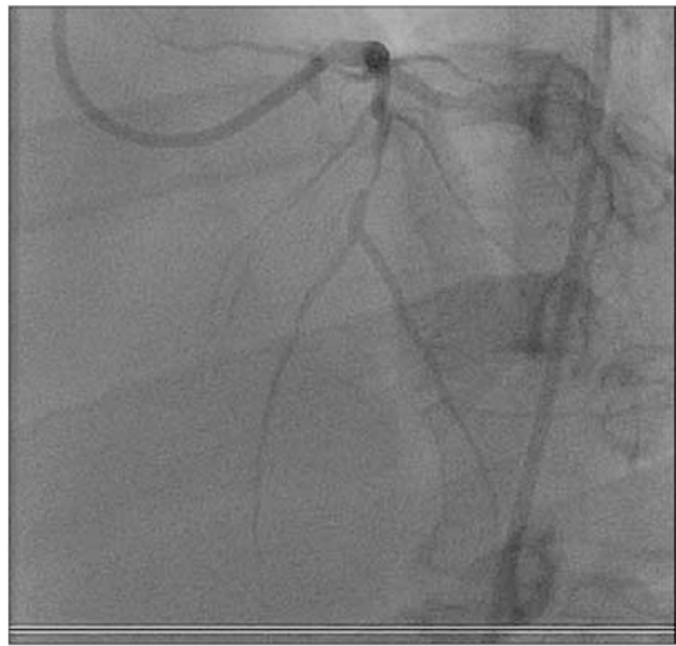

Figure 1 Coronary angiographic image, LAD lesion before intervention.

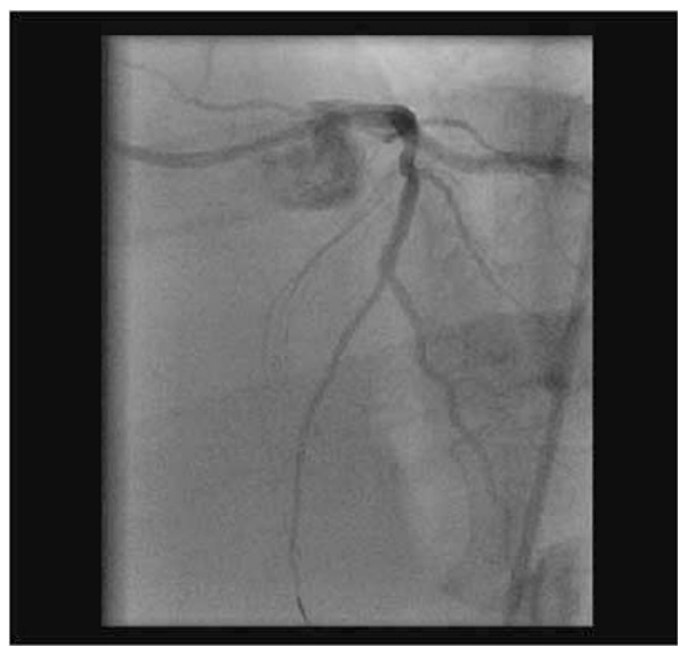

Figure 2 LAD after angioplasty.

dyslipidaemia and renal dysfunction in the syndrome has led to the suggestion that these cardiovascular risk factors may lead to subendocardial infarction with progressive loss of myocardial tissue and function. However there was no evidence of myocardial infarction in affected patients to support this presumption. ${ }^{6}$ Resistance to the action of insulin in the vasculature of ALMS patients contributes to impaired vasodilatation with increased wave reflection and augmented aortic systolic pressure. Such unfavourable central arterial haemodynamics in ALMS may contribute to the development of Alström's cardiomyopathy. ${ }^{9}$ Cardiac magnetic resonance imaging in these patients showed no fluid or fatty infiltration of myocardium, ${ }^{6}$ and all displayed patchy left and right ventricular fibrosis with varying degrees of biventricular impaired function. ${ }^{6}$ However, lack of stress testing in these patients' means that coronary atheroma, which might contribute to cardiac dysfunction cannot be excluded. Because of the young age of presentation of ALMS patients, none of the studies or reports have specifically assessed for the presence of significant ischaemic heart disease with exercise/stress testing or angiography in patients with cardiac problems. Other possibility is atypical ways of presentation, secondary to long standing diabetes and metabolic consequences; thus failing to catch the attention for the CAD. Our index patient, however, presented with classical symptoms, including chest tightness and shortness of breath on exertion.

Coronary atherosclerosis is a chronic process that begins during adolescence and slowly progresses throughout life. Independent risk factors include a family history of premature $\mathrm{CAD}$, cigarette smoking, diabetes mellitus, hypertension, hyperlipidemia, sedentary lifestyle and obesity. These risk factors accelerate or modify a complex and chronic inflammatory process that ultimately manifests as fibro-fatty atherosclerotic plaque. The combination of mixed dyslipidemia, insulin resistance, diabetes, renal impairment and hypertension is very strongly linked to premature atherosclerosis, especially CAD in the general population. This set of disorders is known to occur frequently in Alström subjects. We suggest that Alström subjects should undergo assessment for inducible myocardial ischaemia, even in the absence of classical symptoms.

No clear genotype phenotype correlation has emerged in ALMS so far. Our patient is a compound heterozygote for a frame shift mutation (c.10769delC; p.Thr3590LysfsX6) and nonsense mutation (c.11410C > T; p.Arg3804X) in the ALMS1 gene. Research is ongoing to detect genotype-phenotype correlations. No clinical features are specific to distinguish those with or without confirmed mutations. Whether the multiorgan fibrotic changes seen are a direct result of the mutated ALMS1 gene or due to a chronic inflammatory response to cellular insult due to the loss of function of this gene is unknown.

\section{CONCLUSIONS}

ALMS is a rare autosomal recessive disorder resulting from a mutation in the ALMS1 gene. Associated conditions include diabetes, hypertension, dyslipidemia, obesity and renal impairment. A common cardiac presentation is heart failure secondary to development of a dilated cardiomyopathy. Ours is the first report of a premature CAD within ALMS subjects. We suggest that these patients should be assessed for classical coronary risk factors and investigated specifically to exclude $\mathrm{CAD}$, which may also be a factor in impaired ventricular function in older Alström individuals.

\section{CONFLICT OF INTEREST}

The authors declare no conflict of interest.

1 Alström $\mathrm{CH}$, Hallgren $\mathrm{B}$, Nilsson LB, Asander $\mathrm{H}$ : Retinal degeneration combined with obesity, diabetes mellitus and neurogenous deafness. A specific syndrome (not hitherto described) distinct from Laurence-Moon-Beidl syndrome. A clinical endocrinological and genetic examination based on a large pedigree. Acta psychiatr Neurol Scand 1959; 34(Suppl 129): 1-35.

2 Marshall JD, Beck S, Maffei P et al: Alström syndrome. Eur J Hum Genet 2007; 15 : 1193-1202.

3 Marshall JD, Bronson RT, Collin GB et al: New Alström syndrome phenotype based on the evaluation of 182 cases. Arch Intern Med 2005; 165: 675-683.

4 Marshall JD, Hinman EG, Collin GB et al: Spectrum of ALMS1 variants and evaluation of genotype-phenotype correlations in Alström syndrome. Hum Mutat 2007; 28: 1114-1123.

5 Paisey RB, Carey CM, Bower L et al: Hypertriglyceridemia in Alström syndrome: causes and associations in 37 cases. Clin Endocrinol 2004; 60: 228-231.

6 Loudon MA, Bellenger N, Paisey RB, Carey CM: Cardiac myocardial resonance imaging in adults with Alström syndrome. Orphanet J Rare Diseases 2009; 4: 14

7 Makaryus AN, Zubrow ME, Marshall JD, Gillam LD, Mangion JR: Cardiac manifestations of Alström syndrome: echocardiographic findings. J Am Soc Echocardiogr 2007; 20: 1359-1363.

8 Toulany A, Shea S, Warren AE: Doppler tissue, strain, and strain rate imaging in pediatric patients with Alström syndrome: are there regional functional abnormalities? J Am Soc Echocardiogr 2006; 19: 14-20.

9 Smith JC, McDonnel B, Retallick C et al: Is arterial stiffening in Alström Syndrome linked to the development of cardiomyopathy? Eur J Clin invest 2007; 37: 99-105. 